\title{
Multiple Positive Solutions for Quadratic Integral Equations of Fractional Order
}

\author{
Hui-Sheng Ding, ${ }^{1}$ Man-Man Liu, ${ }^{1}$ and Juan J. Nieto ${ }^{2}$ \\ ${ }^{1}$ College of Mathematics and Information Science, Jiangxi Normal University, Nanchang, Jiangxi 330022, China \\ ${ }^{2}$ Departamento de Estadística, Análisis Matemático y Optimización, Facultad de Matemáticas, \\ Universidad de Santiago de Compostela, 15782 Santiago de Compostela, Spain \\ Correspondence should be addressed to Hui-Sheng Ding; dinghs@mail.ustc.edu.cn
}

Received 26 March 2017; Accepted 6 September 2017; Published 15 October 2017

Academic Editor: Hua Su

Copyright (C) 2017 Hui-Sheng Ding et al. This is an open access article distributed under the Creative Commons Attribution License, which permits unrestricted use, distribution, and reproduction in any medium, provided the original work is properly cited.

In this paper, the existence of multiple positive solutions for a class of quadratic integral equation of fractional order is obtained, by utilizing Avery-Henderson and Leggett-Williams multiple fixed point theorems on cones. An example is given to illustrate the applicability of our results. We believe that this is a first result concerning the existence of multiple solutions for such quadratic integral equation of fractional order.

\section{Introduction and Preliminaries}

Recently, there has been great interest for many authors to study quadratic functional integral equations, which has become one of the most attractive and interesting research areas of integral equations and functional integral equations. There is large literature on this topic. We refer the reader to [1-6] for some of very recent results. In fact, as noted in some earlier literature (see, e.g., [5] and references therein), the nonlinear quadratic functional integral equations have been applied to, for example, the theory of radiative transfer, kinetic theory of gases, the theory of neutron transport, the traffic theory, plasma physics, and numerous branches of mathematical physics.

On the other hand, due to the fact that fractional differential and integral equations have recently been extensively applied in various areas of engineering, mathematics, physics and bioengineering, and other applied sciences, there has been a significant development in fractional integral equations in recent years. Many authors especially have focused on the existence and qualitative properties of solutions for quadratic integral equations of fractional order such as

$$
\begin{aligned}
x(t)= & f(t, x(t)) \\
& +g(t, x(t)) \int_{0}^{t} \frac{(t-s)^{\alpha-1}}{\Gamma(\alpha)} h(s, x(s)) d s,
\end{aligned}
$$

$$
\alpha>0,
$$

and several important variants of (1), and obtain substantial results on this topic (cf. [1-3, 5-9]). However, to the best of our knowledge, it seems that there are no results concerning the existence of multiple solutions for (1) and its variants. That is the main goal of this work. It seems that this is a first result concerning the existence of multiple solutions for such quadratic integral equation of fractional order.

Stimulated by the above works, we aim to investigate the existence of multiple positive solutions for the following fractional order quadratic integral equation:

$$
\begin{array}{r}
x(t)=f[t, x(\lambda(t))] \\
+g[t, x(\mu(t))] \int_{0}^{t} \frac{(t-s)^{\alpha-1}}{\Gamma(\alpha)} h[s, x(\nu(s))] d s, \\
t \in[0,1], \alpha>0 .
\end{array}
$$

See Section 2 for the hypotheses on the involved functions. 
Throughout the rest of this paper, if there is no special statement, we denote by $\mathbb{R}$ the set of real numbers, and by $\mathfrak{L i p}\left(\mathbb{R} \times \mathbb{R}, \mathbb{R}^{+}\right)$the set of all functions $f: \mathbb{R} \times \mathbb{R} \rightarrow \mathbb{R}^{+}$ satisfying that there exists a constant $L_{f}>0$ such that

$$
|f(t, x)-f(t, y)| \leq L_{f}|x-y|, \quad t \in \mathbb{R}, x, y \in \mathbb{R} .
$$

Next, let us recall some notations about cones and two fixed point theorem. For more details, we refer the reader to $[10,11]$.

Let $X$ be a real Banach space. A closed convex set $K$ in $X$ is called a cone if the following conditions are satisfied:

(i) If $x \in K$, then $\lambda x \in K$ for any $\lambda \geq 0$;

(ii) If $x \in K$ and $-x \in K$, then $x=0$.

A nonnegative continuous functional $\psi$ is said to be a concave on $K$ if $\psi$ is continuous and

$$
\begin{aligned}
& \psi(\mu x+(1-\mu) y) \geq \mu \psi(x)+(1-\mu) \psi(y), \\
& x, y \in K, \mu \in[0,1] .
\end{aligned}
$$

Letting $c_{1}, c_{2}, c_{3}$ be three positive constants and $\phi$ be a nonnegative continuous functional on $K$, we denote

$$
\begin{aligned}
K_{c_{1}} & =\left\{y \in K:\|y\|<c_{1}\right\}, \\
\overline{K_{c_{1}}} & =\left\{y \in K:\|y\| \leq c_{1}\right\}, \\
K\left(\phi, c_{1}\right) & :=\left\{x \in K: \phi(x)<c_{1}\right\}, \\
\overline{K\left(\phi, c_{1}\right)} & :=\left\{x \in K: \phi(x) \leq c_{1}\right\}, \\
\partial K\left(\phi, c_{1}\right) & :=\left\{x \in K: \phi(x)=c_{1}\right\}, \\
K\left(\phi, c_{2}, c_{3}\right) & =\left\{y \in K: c_{2} \leq \phi(y),\|y\| \leq c_{3}\right\} .
\end{aligned}
$$

In addition, we say that $\phi$ is increasing on $K$ if $\phi(x) \geq \phi(y)$ for all $x, y \in K$ with $x-y \in K$.

The following two theorems are the well-known AveryHenderson multiple fixed point theorem and LeggettWilliams multiple fixed point theorem, respectively.

Lemma 1 (see [10]). Let $K$ be a cone in a real Banach space $X, \alpha$ and $\varphi$ be two increasing, nonnegative, and continuous functionals on $K$, and $\rho$ be a nonnegative continuous functional on $K$ with $\rho(0)=0$ such that, for some $c>0$ and $M>0$,

$$
\begin{aligned}
\varphi(x) & \leq \rho(x) \leq \alpha(x), \\
\|x\| & \leq M \varphi(x),
\end{aligned}
$$

$$
x \in \overline{K(\varphi, c)} .
$$

Moreover, suppose that there exists a completely continuous operator $\Phi: \overline{K(\varphi, c)} \rightarrow K$ and $0<a<b<c$ such that

$$
\rho(\lambda x) \leq \lambda \rho(x), \quad 0 \leq \lambda \leq 1, x \in \partial K(\rho, b),
$$

and

(i) $\varphi(\Phi x)>c$, for all $x \in \partial K(\varphi, c)$;

(ii) $\rho(\Phi x)<b$, for all $x \in \partial K(\rho, b)$;

(iii) $K(\alpha, a) \neq \emptyset$, and $\alpha(\Phi x)>a$, for all $x \in \partial K(\alpha, a)$.

Then $\Phi$ has at least two fixed points $x_{1}, x_{2}$ belonging to $\overline{K(\varphi, c)}$ such that

$$
\begin{aligned}
a & <\alpha\left(x_{1}\right), \\
\rho\left(x_{1}\right) & <b, \\
b & <\rho\left(x_{2}\right), \\
\varphi\left(x_{2}\right) & <c .
\end{aligned}
$$

Lemma 2 (see [11]). Let $K$ be a cone in a real Banach space $X, c_{4}$ be a positive constant, $\Phi: \overline{K_{c_{4}}} \rightarrow \overline{K_{c_{4}}}$ be a completely continuous mapping, and $\psi$ be a concave nonnegative continuous functional on $K$ with $\psi(u) \leq\|u\|$ for all $u \in \overline{K_{c_{4}}}$. Suppose that there exist three constants $c_{1}, c_{2}, c_{3}$ with $0<c_{1}<c_{2}<c_{3} \leq c_{4}$ such that

(i) $\left\{u \in K\left(\psi, c_{2}, c_{3}\right): \psi(u)>c_{2}\right\} \neq \varnothing$, and $\psi(\Phi u)>c_{2}$ for all $u \in K\left(\psi, c_{2}, c_{3}\right)$;

(ii) $\|\Phi u\|<c_{1}$ for all $u \in \overline{K_{c_{1}}}$;

(iii) $\psi(\Phi u)>c_{2}$ for all $u \in K\left(\psi, c_{2}, c_{4}\right)$ with $\|\Phi u\|>c_{3}$.

Then $\Phi$ has at least three fixed points $u_{1}, u_{2}, u_{3}$ in $\overline{K_{c_{4}}}$. Furthermore, $\left\|u_{1}\right\|<c_{1}<\left\|u_{2}\right\|$, and $\psi\left(u_{2}\right)<c_{2}<\psi\left(u_{3}\right)$.

\section{Main Results}

Firstly, we list some assumptions:

(H1) $f, g, h \in C\left([0,1] \times \mathbb{R}^{+}, \mathbb{R}^{+}\right)$and $f, g \in \mathfrak{Q i \mathfrak { p }}\left(\mathbb{R} \times \mathbb{R}, \mathbb{R}^{+}\right)$.

(H2) $\lambda, \mu, \nu \in C^{1}\left([0,1], \mathbb{R}^{+}\right)$. Moreover, $\lambda^{\prime}(t), \mu^{\prime}(t)>0$ and $\nu^{\prime}(t)>1$.

(H3) $\inf _{t \in[0,1], x \geq 0} f(t, x)>0, \sup _{t \in[0,1], x \geq 0} f(t, x)<+\infty$, $\sup _{t \in[0,1], x \geq 0} g(t, x)<+\infty$, and

$$
\lim _{r \rightarrow+\infty} \frac{\sup _{t \in[0,1], x \in[0, r]} h(t, x)}{r}=0 .
$$

(H4) There exist $0<b<c$ such that

$$
\begin{aligned}
& \inf _{x \geq 0} f(1, x)+\inf _{x \geq 0} g(1, x) \inf _{\nu^{-1}(1) \leq s \leq 1} h(s, c) \\
& . \frac{\left[1-v^{-1}(1)\right]^{\alpha}}{\alpha \Gamma(\alpha)}>c, \\
& \sup _{t \in[0,1], x \geq 0} f(t, x) \\
& \quad+\frac{\sup _{t \in[0,1], x \geq 0} g(t, x) \sup _{t \in[0,1], x \in[0, b]} h(t, x)}{\alpha \Gamma(\alpha)}<b .
\end{aligned}
$$


By a solution of (2) we mean a function $x \in C[0, T]$ satisfying the equation, where

$$
T=\max \left\{\max _{t \in[0,1]} \lambda(t), \max _{t \in[0,1]} \mu(t), \max _{t \in[0,1]} \nu(t)\right\} .
$$

Now, we are ready to present our main result.

Theorem 3. Let (H1)-(H4) hold. Then, there exists $L^{*}>0$ such that (2) has at least two nonnegative solutions provided that $L_{f}<1$ and $L_{g}<L^{*}$.

Proof. By using (H3), we can choose $\sigma \in(0,1)$ satisfying

$$
\begin{aligned}
& \inf _{t \in[0,1], x \geq 0} f(t, x) \geq \sigma\left(\sup _{t \in[0,1], x \geq 0} f(t, x)\right. \\
& \left.+\frac{\sup _{t \in[0,1], x \geq 0} g(t, x) \cdot \sup _{t \in[0,1], x \in[0, c / \sigma]} h(t, x)}{\alpha \Gamma(\alpha)}\right) .
\end{aligned}
$$

Let

$$
\begin{aligned}
K & =\{x \in C[0, T]: x(t)=x(1) \text { for every } t \\
& \left.\in[1, T], \min _{t \in[0,1]} x(t) \geq \sigma\|x\|\right\} .
\end{aligned}
$$

It is not difficult to verify that $K$ is a cone in $C[0, T]$. Let

$$
\begin{aligned}
& \varphi(u)=u(1), \\
& \rho(u)=\alpha(u)=\|u\|=\max _{t \in[0, T]} u(t)=\max _{t \in[0,1]} u(t),
\end{aligned}
$$

$u \in K$.

Obviously, $\varphi, \rho$, and $\alpha$ are increasing, nonnegative, and continuous functionals on $K$ with $\rho(0)=0$. Moreover, we have

$$
\begin{aligned}
\|u\| & \leq \frac{1}{\sigma} \min _{t \in[0,1]} u(t) \leq \sigma^{-1} \varphi(u), \\
\rho(\lambda u) & =\lambda \rho(u),
\end{aligned}
$$

for all $u \in K$ and $0 \leq \lambda \leq 1$. We divide the remaining proof by three steps.

Step 1. Let

$\Omega$ $=\{x \in C[0, T]: x(t)=x(1)$ for every $t \in[1, T]\}$.

For every $y \in \overline{K(\varphi, c)}$, define an operator $A_{y}$ on $\Omega$ by

$$
\left(A_{y} x\right)(t)= \begin{cases}f[t, x(\lambda(t))]+g[t, x(\mu(t))] \int_{0}^{t} \frac{(t-s)^{\alpha-1}}{\Gamma(\alpha)} h[s, y(\nu(s))] d s, & x \in \Omega, t \in[0,1], \\ \left(A_{y} x\right)(1), & x \in \Omega, t \in[1, T] .\end{cases}
$$

It is easy to see that $A_{y}(\Omega) \subset \Omega$.

For $x, z \in \Omega$ and $t \in[0,1]$, there holds

$$
\begin{aligned}
& \left|\left(A_{y} x\right)(t)-\left(A_{y} z\right)(t)\right| \\
& \leq|f[t, x(\lambda(t))]-f[t, z(\lambda(t))]| \\
& \quad+|g[t, x(\mu(t))]-g[t, z(\mu(t))]| \\
& \quad \cdot\left|\int_{0}^{t} \frac{(t-s)^{\alpha-1}}{\Gamma(\alpha)} h[s, y(\nu(s))] d s\right| \\
& \leq L_{f}\|x-z\| \\
& \quad+\frac{L_{g} \cdot \sup _{t \in[0,1], x \in[0, c / \sigma]} h(t, x)}{\alpha \Gamma(\alpha)}\|x-z\|,
\end{aligned}
$$

which yields that

$$
\left\|A_{y} x-A_{y} z\right\|=\max _{t \in[0, T]}\left|\left(A_{y} x\right)(t)-\left(A_{y} z\right)(t)\right|
$$

$$
\begin{aligned}
= & \max _{t \in[0,1]}\left|\left(A_{y} x\right)(t)-\left(A_{y} z\right)(t)\right| \\
\leq & L_{f}\|x-z\| \\
& +\frac{L_{g} \cdot \sup _{t \in[0,1], x \in[0, c / \sigma]} h(t, x)}{\alpha \Gamma(\alpha)}\|x-z\| .
\end{aligned}
$$

Let

$$
L^{*}=\frac{\alpha \Gamma(\alpha)\left(1-L_{f}\right)}{\sup _{t \in[0,1], x \in[0, c / \sigma]} h(t, x)} .
$$

Then, noting that $L_{f}<1, A_{y}$ has a unique fixed point $x_{y} \in \Omega$ provided that $L_{g}<L^{*}$.

Step 2. Now, define an operator $A$ on $\overline{K(\varphi, c)}$ by

$$
(A y)(t)=x_{y}(t)= \begin{cases}f\left[t, x_{y}(\lambda(t))\right]+g\left[t, x_{y}(\mu(t))\right] \int_{0}^{t} \frac{(t-s)^{\alpha-1}}{\Gamma(\alpha)} h[s, y(\nu(s))] d s, & y \in \overline{K(\varphi, c)}, t \in[0,1] \\ (A y)(1), & y \in \overline{K(\varphi, c)}, t \in[1, T] .\end{cases}
$$


Noting that, for every $t \in[0,1]$ and $y \in \overline{K(\varphi, c)}$, by (12), we have

$$
\begin{aligned}
& (A y)(t) \geq \inf _{t \in[0,1], x \geq 0} f(t, x) \geq \sigma\left(\sup _{t \in[0,1], x \geq 0} f(t, x)\right. \\
& \left.+\frac{\sup _{t \in[0,1], x \geq 0} g(t, x) \cdot \sup _{t \in[0,1], x \in[0, c / \sigma]} h(t, x)}{\alpha \Gamma(\alpha)}\right) \\
& \geq \sigma\|A y\|,
\end{aligned}
$$

which yields that $A(\overline{K(\varphi, c)}) \subset K$.

Next, let us show that $A: \overline{K(\varphi, c)} \rightarrow K$ is completely continuous. Let $y_{n} \rightarrow y$ in $\overline{K(\varphi, c)}$. For $t \in[0,1]$, we have

$$
\begin{gathered}
\left|\left(A y_{n}\right)(t)-(A y)(t)\right|=\mid f\left[t,\left(A y_{n}\right)(\lambda(t))\right]-f[t, \\
(A y)(\lambda(t))]|+| g\left[t,\left(A y_{n}\right)(\mu(t))\right] \\
\cdot \int_{0}^{t} \frac{(t-s)^{\alpha-1}}{\Gamma(\alpha)} h\left[s, y_{n}(\nu(s))\right] d s-g[t,(A y) \\
\cdot(\mu(t))] \int_{0}^{t} \frac{(t-s)^{\alpha-1}}{\Gamma(\alpha)} h[s, y(\nu(s))] d s \mid
\end{gathered}
$$

$$
\begin{aligned}
& \leq L_{f}\left|\left(A y_{n}\right)(\lambda(t))-(A y)(\lambda(t))\right| \\
& +\frac{L_{g} \cdot \sup _{t \in[0,1], x \in[0, c / \sigma]} h(t, x)}{\alpha \Gamma(\alpha)} \mid\left(A y_{n}\right)(\mu(t)) \\
& -(A y)(\mu(t)) \mid+\sup _{t \in[0,1], x \geq 0} g(t, x) \\
& \cdot \int_{0}^{t} \frac{(t-s)^{\alpha-1}}{\Gamma(\alpha)} \mid h\left[s, y_{n}(\nu(s))\right]
\end{aligned}
$$$$
-h[s, y(\nu(s))] \mid d s \leq\left(L_{f}\right.
$$$$
\left.+\frac{L_{g} \cdot \sup _{t \in[0,1], x \in[0, c / \sigma]} h(t, x)}{\alpha \Gamma(\alpha)}\right)\left\|A y_{n}-A y\right\|
$$$$
+\sup _{t \in[0,1], x \geq 0} g(t, x) \int_{0}^{t} \frac{(t-s)^{\alpha-1}}{\Gamma(\alpha)} \mid h\left[s, y_{n}(\nu(s))\right]
$$$$
-h[s, y(\nu(s))] \mid d s,
$$

which yields that

$$
\begin{aligned}
\left\|A y_{n}-A y\right\| & =\max _{t \in[0, T]}\left|\left(A y_{n}\right)(t)-(A y)(t)\right|=\max _{t \in[0,1]}\left|\left(A y_{n}\right)(t)-(A y)(t)\right| \\
& \leq \frac{\alpha \Gamma(\alpha) \sup _{t \in[0,1], x \geq 0} g(t, x) \cdot \sup _{t \in[0,1]} \int_{0}^{t}\left((t-s)^{\alpha-1} / \Gamma(\alpha)\right)\left|h\left[s, y_{n}(\nu(s))\right]-h[s, y(\nu(s))]\right| d s}{\alpha \Gamma(\alpha)-\alpha \Gamma(\alpha) L_{f}-L_{g} \sup _{t \in[0,1], x \in[0, c / \sigma]} h(t, x)},
\end{aligned}
$$

where

$$
\alpha \Gamma(\alpha)>\alpha \Gamma(\alpha) L_{f}+L_{g} \sup _{t \in[0,1], x \in[0, c / \sigma]} h(t, x)
$$

since $L_{g}<L^{*}$. Combining (24) with the fact $h$ is uniformly continuous on $[0,1] \times[0, c / \sigma]$ and $y_{n}(t)$ uniformly converges to $y(t)$ on $[0, T]$, we conclude that $A y_{n} \rightarrow A y$ in $K$. It suffices to show $A(\overline{K(\varphi, c)})$ is precompact. It follows from the above proof that $\{(A y)(t): y \in \overline{K(\varphi, c)}\}$ is uniformly bounded on $[0, T]$. For every $y \in \overline{K(\varphi, c)}$ and $t_{1}, t_{2} \in[0,1]$ with $t_{1} \leq t_{2}$, we have

$$
\begin{aligned}
& \left|(A y)\left(t_{1}\right)-(A y)\left(t_{2}\right)\right| \leq \mid f\left[t_{1},(A y)\left(\lambda\left(t_{1}\right)\right)\right] \\
& -f\left[t_{2},(A y)\left(\lambda\left(t_{2}\right)\right)\right]|+| g\left[t_{1},(A y)\left(\mu\left(t_{1}\right)\right)\right] \\
& \quad \cdot \int_{0}^{t_{1}} \frac{\left(t_{1}-s\right)^{\alpha-1}}{\Gamma(\alpha)} h[s, y(\nu(s))] d s \\
& -g\left[t_{2},(A y)\left(\mu\left(t_{2}\right)\right)\right]
\end{aligned}
$$

$$
\begin{aligned}
& \cdot \int_{0}^{t_{2}} \frac{\left(t_{2}-s\right)^{\alpha-1}}{\Gamma(\alpha)} h[s, y(\nu(s))] d s \mid \\
& \leq\left|f\left[t_{1},(A y)\left(\lambda\left(t_{1}\right)\right)\right]-f\left[t_{2},(A y)\left(\lambda\left(t_{1}\right)\right)\right]\right| \\
& +L_{f}\left|(A y)\left(\lambda\left(t_{1}\right)\right)-(A y)\left(\lambda\left(t_{2}\right)\right)\right| \\
& +\frac{L_{g} \cdot \sup _{t \in[0,1], x \in[0, c / \sigma]} h(t, x)}{\alpha \Gamma(\alpha)} \mid(A y)\left(\mu\left(t_{1}\right)\right) \\
& -(A y)\left(\mu\left(t_{2}\right)\right) \mid \\
& +\frac{\sup _{t \in[0,1], x \in[0, c / \sigma]} h(t, x)}{\alpha \Gamma(\alpha)} \mid g\left[t_{1},(A y)\left(\mu\left(t_{1}\right)\right)\right] \\
& -g\left[t_{2},(A y)\left(\mu\left(t_{1}\right)\right)\right] \mid+\sup _{t \in[0,1], x \geq 0} g(t, x) \\
& \cdot \sup _{t \in[0,1], x \in[0, c / \sigma]} h(t, x) \\
& \cdot\left|\int_{0}^{t_{1}} \frac{\left(t_{1}-s\right)^{\alpha-1}}{\Gamma(\alpha)} d s-\int_{0}^{t_{2}} \frac{\left(t_{2}-s\right)^{\alpha-1}}{\Gamma(\alpha)} d s\right| .
\end{aligned}
$$


On the other hand, we have

$$
\begin{gathered}
\left|\int_{0}^{t_{1}} \frac{\left(t_{1}-s\right)^{\alpha-1}}{\Gamma(\alpha)} d s-\int_{0}^{t_{2}} \frac{\left(t_{2}-s\right)^{\alpha-1}}{\Gamma(\alpha)} d s\right| \\
\leq \int_{0}^{t_{1}}\left|\frac{\left(t_{1}-s\right)^{\alpha-1}}{\Gamma(\alpha)}-\frac{\left(t_{2}-s\right)^{\alpha-1}}{\Gamma(\alpha)}\right| d s \\
\quad+\int_{t_{1}}^{t_{2}} \frac{\left(t_{2}-s\right)^{\alpha-1}}{\Gamma(\alpha)} d s \\
\leq \frac{1}{\Gamma(\alpha)} \int_{0}^{t_{1}}\left|s^{\alpha-1}-\left(t_{2}-t_{1}+s\right)^{\alpha-1}\right| d s \\
\quad+\frac{1}{\alpha \Gamma(\alpha)}\left(t_{2}-t_{1}\right)^{\alpha} .
\end{gathered}
$$

By Lebesgue's dominated convergence theorem, as $t_{2}-t_{1} \rightarrow 0$,

$$
\begin{aligned}
& \int_{0}^{t_{1}}\left|s^{\alpha-1}-\left(t_{2}-t_{1}+s\right)^{\alpha-1}\right| d s \\
& \quad \leq \int_{0}^{1}\left|s^{\alpha-1}-\left(t_{2}-t_{1}+s\right)^{\alpha-1}\right| d s \longrightarrow 0 .
\end{aligned}
$$

Combining this with the fact that $f, g$ are uniformly continuous on compact sets, we conclude that, for every $\varepsilon>0$, there exists $\delta^{\prime}>0$ such that, for all $t_{1}, t_{2} \in[0,1]$ with $t_{1} \leq t_{2}$ and $\left|t_{1}-t_{2}\right|<\delta^{\prime}$, and $y \in \overline{K(\varphi, c)}$, there hold

$$
\begin{aligned}
& \left|f\left[t_{1},(A y)\left(\lambda\left(t_{1}\right)\right)\right]-f\left[t_{2},(A y)\left(\lambda\left(t_{1}\right)\right)\right]\right|<\frac{\varepsilon}{3}, \\
& \frac{\sup _{t \in[0,1], x \in[0, c / \sigma]} h(t, x)}{\alpha \Gamma(\alpha)} \mid g\left[t_{1},(A y)\left(\mu\left(t_{1}\right)\right)\right] \\
& \quad-g\left[t_{2},(A y)\left(\mu\left(t_{1}\right)\right)\right] \mid<\frac{\varepsilon}{3}, \\
& \sup _{t \in[0,1], x \geq 0} g(t, x) \cdot \sup _{t \in[0,1], x \in[0, c / \sigma]} h(t, x) \\
& \quad\left|\int_{0}^{t_{1}} \frac{\left(t_{1}-s\right)^{\alpha-1}}{\Gamma(\alpha)} d s-\int_{0}^{t_{2}} \frac{\left(t_{2}-s\right)^{\alpha-1}}{\Gamma(\alpha)} d s\right|<\frac{\varepsilon}{3} .
\end{aligned}
$$

Thus, we conclude that, for every $\varepsilon>0$,

$$
\begin{aligned}
& \left|(A y)\left(t_{1}\right)-(A y)\left(t_{2}\right)\right| \leq L_{f} \mid(A y)\left(\lambda\left(t_{1}\right)\right) \\
& -(A y)\left(\lambda\left(t_{2}\right)\right) \mid \\
& +\frac{L_{g} \cdot \sup _{t \in[0,1], x \in[0, c / \sigma]} h(t, x)}{\alpha \Gamma(\alpha)} \mid(A y)\left(\mu\left(t_{1}\right)\right) \\
& -(A y)\left(\mu\left(t_{2}\right)\right) \mid+\varepsilon
\end{aligned}
$$

holds for all $t_{1}, t_{2} \in[0,1]$ with $t_{1} \leq t_{2}$ and $\left|t_{1}-t_{2}\right|<\delta^{\prime}$, and $y \in \overline{K(\varphi, c)}$, which yields that, for all $t_{1}, t_{2} \in[0,1]$ with $t_{1} \leq t_{2}$ and $\left|t_{1}-t_{2}\right|<\delta^{\prime}$, there holds

$$
\begin{aligned}
& \sup _{y \in \overline{K(\varphi, c)}}\left|(A y)\left(t_{1}\right)-(A y)\left(t_{2}\right)\right| \\
& \leq L_{f} \cdot \sup _{y \in \overline{K(\varphi, c)}}\left|(A y)\left(\lambda\left(t_{1}\right)\right)-(A y)\left(\lambda\left(t_{2}\right)\right)\right| \\
& +\frac{L_{g} \cdot \sup _{t \in[0,1], x \in[0, c / \sigma]} h(t, x)}{\alpha \Gamma(\alpha)} \\
& \quad \cdot \sup _{y \in \overline{K(\varphi, c)}}\left|(A y)\left(\mu\left(t_{1}\right)\right)-(A y)\left(\mu\left(t_{2}\right)\right)\right|+\varepsilon .
\end{aligned}
$$

Then, we have

$$
\begin{aligned}
& \left.\lim _{t_{2}-t_{1} \rightarrow 0} \sup _{y \in \overline{K(\varphi, c)}}\left|(A y)\left(t_{1}\right)-(A y)\left(t_{2}\right)\right|\right) \leq L_{f} \\
& \left.\cdot \lim _{t_{2}-t_{1} \rightarrow 0} \sup _{y \in \overline{K(\varphi, c)}}\left|(A y)\left(\lambda\left(t_{1}\right)\right)-(A y)\left(\lambda\left(t_{2}\right)\right)\right|\right) \\
& +\frac{L_{g} \cdot \sup _{t \in[0,1], x \in[0, c / \sigma]} h(t, x)}{\alpha \Gamma(\alpha)} \\
& \left.\cdot \lim _{t_{2}-t_{1} \rightarrow 0} \sup _{y \in \overline{K(\varphi, c)}}\left|(A y)\left(\mu\left(t_{1}\right)\right)-(A y)\left(\mu\left(t_{2}\right)\right)\right|\right) \\
& +\varepsilon,
\end{aligned}
$$

where

$$
\begin{aligned}
& \limsup _{t_{2}-t_{1} \rightarrow 0}\left(\sup _{y \in \overline{K(\varphi, c)}}\left|(A y)\left(t_{1}\right)-(A y)\left(t_{2}\right)\right|\right) \\
& \quad:=\inf _{\delta>0} \sup _{0 \leq t_{1} \leq t_{2} \leq t_{1}+\delta \leq 1}\left(\sup _{y \in \overline{K(\varphi, c)}}\left|(A y)\left(t_{1}\right)-(A y)\left(t_{2}\right)\right|\right), \\
& \limsup _{t_{2}-t_{1} \rightarrow 0}\left(\sup _{y \in \overline{K(\varphi, c)}}\left|(A y)\left(\lambda\left(t_{1}\right)\right)-(A y)\left(\lambda\left(t_{2}\right)\right)\right|\right), \\
& \limsup _{t_{2}-t_{1} \rightarrow 0}\left(\sup _{y \in \overline{K(\varphi, c)}}\left|(A y)\left(\mu\left(t_{1}\right)\right)-(A y)\left(\mu\left(t_{2}\right)\right)\right|\right)
\end{aligned}
$$

are similarly defined. Noting that $\lambda^{\prime}(t), \mu^{\prime}(t)>0$ for all $t \in$ $[0,1]$, we conclude

$$
\begin{aligned}
& \limsup _{t_{2}-t_{1} \rightarrow 0}\left(\sup _{y \in \overline{K(\varphi, c)}}\left|(A y)\left(t_{1}\right)-(A y)\left(t_{2}\right)\right|\right) \\
& =\limsup _{t_{2}-t_{1} \rightarrow 0}\left(\sup _{y \in \overline{K(\varphi, c)}}\left|(A y)\left(\lambda\left(t_{1}\right)\right)-(A y)\left(\lambda\left(t_{2}\right)\right)\right|\right) \\
& =\limsup _{t_{2}-t_{1} \rightarrow 0}\left(\sup _{y \in \overline{K(\varphi, c)}}\left|(A y)\left(\mu\left(t_{1}\right)\right)-(A y)\left(\mu\left(t_{2}\right)\right)\right|\right),
\end{aligned}
$$


and so we have

$$
\begin{aligned}
& \lim _{t_{2}-t_{1} \rightarrow 0}\left(\sup _{y \in \overline{K(\varphi, c)}}\left|(A y)\left(t_{1}\right)-(A y)\left(t_{2}\right)\right|\right) \\
& \leq \frac{\alpha \Gamma(\alpha) \varepsilon}{\alpha \Gamma(\alpha)-\alpha \Gamma(\alpha) L_{f}-L_{g} \sup _{t \in[0,1], x \in[0, c / \sigma]} h(t, x)} .
\end{aligned}
$$

Thus, $\{(A y)(t): y \in \overline{K(\varphi, c)}\}$ is equicontinuous on $[0,1]$. Then, it is not difficult to obtain that $\{(A y)(t): y \in \overline{K(\varphi, c)}\}$ is equicontinuous on $[0, T]$. This proves that $A(\overline{K(\varphi, c)})$ is precompact, and thus $A: \overline{K(\varphi, c)} \rightarrow K$ is completely continuous.

Step 3. It remains to verify the assumptions (i)-(iii) of Lemma 1 . For every $y \in \partial K(\varphi, c)$, noting that $y(1)=c$ and $y(t)=c$ for all $t \in[1, T]$, by (H4), we have

$$
\begin{aligned}
& \varphi(A y)=(A y)(1)=f[1,(A y)(\lambda(1))] \\
& +g[1,(A y)(\mu(1))] \int_{0}^{1} \frac{(1-s)^{\alpha-1}}{\Gamma(\alpha)} h[s, y(\nu(s))] d s \\
& \quad \geq \inf _{x \geq 0} f(1, x) \\
& +\inf _{x \geq 0} g(1, x) \int_{v^{-1}(1)}^{1} \frac{(1-s)^{\alpha-1}}{\Gamma(\alpha)} h(s, c) d s \\
& \geq \inf _{x \geq 0} f(1, x)+\inf _{x \geq 0} g(1, x) \inf _{v^{-1}(1) \leq s \leq 1} h(s, c) \\
& \quad . \frac{\left[1-v^{-1}(1)\right]^{\alpha}}{\alpha \Gamma(\alpha)}>c .
\end{aligned}
$$

For every $y \in \partial K(\rho, b)$, noting that $y(t) \leq b$ for all $t \in[0, T]$, again by $(\mathrm{H} 4)$, we have

$$
\begin{aligned}
& \rho(A y)=\sup _{t \in[0,1]}\{f[t,(A y)(\lambda(t))] \\
& +g[t,(A y)(\mu(t))] \\
& \left.\quad \cdot \int_{0}^{t} \frac{(t-s)^{\alpha-1}}{\Gamma(\alpha)} h[s, y(\nu(s))] d s\right\} \\
& \leq \sup _{t \in[0,1]}\left\{\sup _{t \in[0,1], x \geq 0} f(t, x)+\sup _{t \in[0,1], x \geq 0} g(t, x)\right. \\
& \left.\quad \cdot \sup _{t \in[0,1], x \in[0, b]} h(t, x) \int_{0}^{t} \frac{(t-s)^{\alpha-1}}{\Gamma(\alpha)} d s\right\} \\
& \leq \sup _{t \in[0,1], x \geq 0} f(t, x) \\
& \quad+\frac{\sup _{t \in[0,1], x \geq 0} g(t, x) \sup _{t \in[0,1], x \in[0, b]} h(t, x)}{\alpha \Gamma(\alpha)}<b .
\end{aligned}
$$

In addition, letting

$$
a=\frac{1}{2} \min \left\{\inf _{t \in[0,1], x \geq 0} f(t, x), b\right\},
$$

it is easy to see that the assumption (iii) of Lemma 1 holds.
Then, by Lemma 1 , we conclude that $A$ has at least two fixed points $x_{1}, x_{2} \in \overline{K(\varphi, c)}$. Thus,

$$
\begin{aligned}
& x_{i}(t) \\
& =f\left[t, x_{i}(\lambda(t))\right] \\
& +g\left[t, x_{i}(\mu(t))\right] \int_{0}^{t} \frac{(t-s)^{\alpha-1}}{\Gamma(\alpha)} h\left[s, x_{i}(\nu(s))\right] d s, \\
& \quad t \in[0,1], i=1,2 ;
\end{aligned}
$$

that is, $x_{1}, x_{2}$ are two nonnegative solutions for (2).

Remark 4. Due to the influence of fractional term $(t-s)^{\alpha-1}$ in (2), it becomes more difficult to verify the assumptions of Lemma 1. In addition, we have tried to consider the case of $v^{\prime}(t) \leq 1$, where it seems that the approach used here can not be applied to study (2). We leave it for further research.

By using Lemma 2, we can obtain the existence of three nonnegative solutions to (2).

Corollary 5. Let (H1)-(H4) hold and the first inequality in (H4) is strengthened to

$$
\begin{gathered}
\inf _{x \geq 0} f(1, x)+\inf _{x \geq 0} g(1, x) \inf _{v^{-1}(1) \leq s \leq 1, x \geq c} h(s, x) \\
\cdot \frac{\left[1-v^{-1}(1)\right]^{\alpha}}{\alpha \Gamma(\alpha)}>c .
\end{gathered}
$$

Then, there exists $L_{*}>0$ such that (2) has at least three nonnegative solutions provided that $L_{f}<1$ and $L_{g}<L_{*}$.

Proof. By using (H3), we can choose $\rho \in(0,1)$ such that

$$
\begin{aligned}
& \inf _{t \in[0,1], x \geq 0} f(t, x) \geq \rho\left(\sup _{t \in[0,1], x \geq 0} f(t, x)\right. \\
& \left.+\frac{\sup _{t \in[0,1], x \geq 0} g(t, x) \cdot \sup _{t \in[0,1], x \in[0, c / \rho]} h(t, x)}{\alpha \Gamma(\alpha)}\right), \\
& \sup _{t \in[0,1], x \geq 0} f(t, x) \\
& +\frac{\sup _{t \in[0,1], x \geq 0} g(t, x) \sup _{t \in[0,1], x \in[0, c / \rho]} h(t, x)}{\alpha \Gamma(\alpha)} \leq \frac{c}{\rho} .
\end{aligned}
$$

Let $T$ be the same as in Theorem 3 and

$$
\begin{aligned}
K & =\{x \in C[0, T]: x(t)=x(1) \text { for every } t \\
& \left.\in[1, T], \min _{t \in[0,1]} x(t) \geq \rho\|x\|\right\} .
\end{aligned}
$$


Moreover, denote $\Omega$ and $A_{y}\left(y \in \overline{K_{c_{4}}}\right)$ be the same as in Theorem 3, and

$$
\begin{aligned}
& c_{1}=b, \\
& c_{2}=c, \\
& c_{3}=c_{4}=\frac{c}{\rho} .
\end{aligned}
$$

Then, by a similar proof to Step 1 of Theorem 3, we can prove that $A_{y}$ has a unique fixed point $x_{y} \in \Omega$ provided that $L_{g}<$ $L_{*}$, where

$$
L_{*}=\frac{\alpha \Gamma(\alpha)\left(1-L_{f}\right)}{\sup _{t \in[0,1], x \in[0, c / \rho]} h(t, x)} .
$$

Now, we can define

$$
(A y)(t)=x_{y}(t)= \begin{cases}f\left[t, x_{y}(\lambda(t))\right]+g\left[t, x_{y}(\mu(t))\right] \int_{0}^{t} \frac{(t-s)^{\alpha-1}}{\Gamma(\alpha)} h[s, y(\nu(s))] d s, \quad y \in \overline{K_{c_{4}}}, t \in[0,1] \\ (A y)(1), & y \in \overline{K_{c_{4}}}, t \in[1, T]\end{cases}
$$

Analogously to the proof of Theorem 3, we can also show that $A: \overline{K_{c_{4}}} \rightarrow K$ is completely continuous. Let

$$
\psi(u)=u(1), \quad u \in K
$$

Obviously, $\psi$ is a concave nonnegative continuous functional on $K$ and $\psi(u) \leq\|u\|$. For every $y \in \overline{K_{c_{4}}}$, by (42), we have

$$
\begin{aligned}
& \|A y\|=\sup _{t \in[0,1]}\left\{f\left[t, x_{y}(\lambda(t))\right]+g\left[t, x_{y}(\mu(t))\right]\right. \\
& \left.\cdot \int_{0}^{t} \frac{(t-s)^{\alpha-1}}{\Gamma(\alpha)} h[s, y(\nu(s))] d s\right\} \\
& \leq \sup _{t \in[0,1]}\left\{\sup _{t \in[0,1], x \geq 0} f(t, x)+\sup _{t \in[0,1], x \geq 0} g(t, x)\right. \\
& \left.\cdot \sup _{t \in[0,1], x \in[0, c / \rho]} h(t, x) \int_{0}^{t} \frac{(t-s)^{\alpha-1}}{\Gamma(\alpha)} d s\right\} \\
& \leq \sup _{t \in[0,1], x \geq 0} f(t, x) \\
& +\frac{\sup _{t \in[0,1], x \geq 0} g(t, x) \sup _{t \in[0,1], x \in[0, c / \rho]} h(t, x)}{\alpha \Gamma(\alpha)} \\
& <\frac{c}{\rho}=c_{4}, \quad
\end{aligned}
$$

which means that $A$ maps $\overline{K_{c_{4}}}$ to $\overline{K_{c_{4}}}$. Similarly, by (H4), we have

$$
\begin{aligned}
\|A y\| \leq & \sup _{t \in[0,1], x \geq 0} f(t, x) \\
& +\frac{\sup _{t \in[0,1], x \geq 0} g(t, x) \sup _{t \in[0,1], x \in[0, b]} h(t, x)}{\alpha \Gamma(\alpha)} \\
<b & =c_{1}, \quad y \in \overline{K_{c_{1}}} ;
\end{aligned}
$$

that is, the condition (ii) of Lemma 2 holds. Moreover, the condition (iii) of Lemma 2 follows from the definition of $K$ and $c_{3}$. It remains to show that the condition (i) of Lemma 2 holds. For every $y \in K\left(\psi, c_{2}, c_{3}\right)$, noting that $y(1) \geq c_{2}=c$ and thus $y(t) \geq c$ for all $t \in[1, T]$, by (40), we have

$$
\begin{aligned}
& \psi(A y)=(A y)(1) \\
&= f\left[1, x_{y}(\lambda(1))\right] \\
&+g\left[1, x_{y}(\mu(1))\right] \int_{0}^{1} \frac{(1-s)^{\alpha-1}}{\Gamma(\alpha)} h[s, y(\nu(s))] d s \\
& \geq \inf _{x \geq 0} f(1, x) \\
& \quad+\inf _{x \geq 0} g(1, x) \int_{v^{-1}(1)}^{1} \frac{(1-s)^{\alpha-1}}{\Gamma(\alpha)} \inf _{x \geq c} h(s, x) d s \\
& \geq \inf _{x \geq 0} f(1, x)+\inf _{x \geq 0} g(1, x) \inf _{v^{-1}(1) \leq s \leq 1, x \geq c} h(s, x) \\
& \quad \cdot \frac{\left[1-v^{-1}(1)\right]^{\alpha}}{\alpha \Gamma(\alpha)}>c .
\end{aligned}
$$

Then, by Lemma 2, $A$ has at least three fixed points in $\overline{K_{c_{4}}}$, and thus (2) has at least three nonnegative solutions.

Next, we present an example to show the applicability of our results.

Example 6. For $t \in[0,1]$ and $x \geq 0$, let $\alpha=1 / 2$ :

$$
\begin{aligned}
& \lambda(t)=\mu(t)=t, \\
& v(t)=2 t, \\
& f(t, x)=1+\frac{1}{4} \sin ^{2}(t x), \\
& g(t, x)=1+\beta e^{-t^{2} x^{2}}, \\
& h(t, x) \\
& = \begin{cases}\sqrt{x}, & 0 \leq x \leq 10, \\
\frac{\sqrt{2} \gamma-1}{\sqrt{10}} x+(2-\sqrt{2} \gamma) \sqrt{10}, & 10<x<20, \\
\gamma \sqrt{x}, & x \geq 20,\end{cases}
\end{aligned}
$$

where $\beta, \gamma>0$ are two constants. 
It is not difficult to show that (H1) holds with $L_{f} \leq 1 / 2$ and $L_{g} \leq \beta \sqrt{2} e^{-1 / 2}$. Moreover, it is easy to verify that $(\mathrm{H} 2)$ and (H3) hold. Letting $b=10$ and $c=20$, we have

$$
\begin{aligned}
& \inf _{x \geq 0} f(1, x)+\inf _{x \geq 0} g(1, x) \inf _{\nu^{-1}(1) \leq s \leq 1, x \geq c} h(s, x) \\
& \cdot \frac{\left[1-\nu^{-1}(1)\right]^{\alpha}}{\alpha \Gamma(\alpha)} \geq 1+\gamma \sqrt{20} \cdot \sqrt{\frac{2}{\pi}}=1+\gamma \sqrt{\frac{40}{\pi}} \\
& >20=c,
\end{aligned}
$$

provided that $\gamma>\sqrt{19 \pi / 40}$, and

$$
\begin{aligned}
& \sup _{t \in[0,1], x \geq 0} f(t, x) \\
& +\frac{\sup _{t \in[0,1], x \geq 0} g(t, x) \sup _{t \in[0,1], x \in[0, b]} h(t, x)}{\alpha \Gamma(\alpha)} \leq \frac{5}{4} \\
& +\frac{(1+\beta) \sqrt{10}}{\sqrt{\pi} / 2}<10=b,
\end{aligned}
$$

provided that $\beta<1$. Then, by Corollary 5, (2) has at least three nonnegative solutions provided that $\gamma>\sqrt{19 \pi / 40}$ and $\beta$ is sufficiently small.

\section{Conflicts of Interest}

The authors declare that they have no conflicts of interest.

\section{Acknowledgments}

H.-S. Ding acknowledges support from NSFC (11461034), the NSF of Jiangxi Province (20143ACB21001), and the Foundation of Jiangxi Provincial Education Department (GJJ150342). M. M. Liu acknowledges support from the Graduate Innovation Fund of Jiangxi Province (YC2016S157). The research of J. J. Nieto has been partially supported by AEI and the Ministerio de Economia y Competitividad of Spain under Grant MTM2016-75140-P and XUNTA de Galicia under Grant GRC2015-004 and cofinanced by the European Community fund FEDER.

\section{References}

[1] M. A. Darwish and K. Sadarangani, "On a quadratic integral equation with supremum involving Erdélyi-Kober fractional order," Mathematische Nachrichten, vol. 288, no. 5-6, pp. 566576, 2015.

[2] M. A. Darwish, "On Erdélyi-Kober fractional Urysohn-Volterra quadratic integral equations," Applied Mathematics and Computation, vol. 273, pp. 562-569, 2016.

[3] B. C. Dhage, S. B. Dhage, and J. R. Graef, "Local attractivity and stability analysis of a nonlinear quadratic fractional integral equation," Applicable Analysis, vol. 95, no. 9, pp. 1989-2003, 2016.

[4] A. Khchine, L. Maniar, and M.-A. Taoudi, "Leray-Schaudertype fixed point theorems in Banach algebras and application to quadratic integral equations," Fixed Point Theory and Applications, vol. 2016, article 88, 2016.
[5] M. M. Metwali, "On a class of quadratic Urysohn-Hammerstein integral equations of mixed type and initial value problem of fractional order," Mediterranean Journal of Mathematics, vol. 13, no. 5, pp. 2691-2707, 2016.

[6] L. N. Mishra and M. Sen, "On the concept of existence and local attractivity of solutions for some quadratic Volterra integral equation of fractional order," Applied Mathematics and Computation, vol. 285, pp. 174-183, 2016.

[7] S. Abbas, M. Benchohra, M. Rivero, and J. J. Trujillo, "Existence and stability results for nonlinear fractional order Riemann-Liouville Volterra-Stieltjes quadratic integral equations," Applied Mathematics and Computation, vol. 247, pp. 319328, 2014.

[8] B. C. Dhage and N. K. Sotiris, "Existence of positive monotonic solutions of functional hybrid fractional integral equations of quadratic type," Fixed Point Theory, vol. 16, no. 2, pp. 273-284, 2015.

[9] A. El-Sayed and H. Hashem, "Existence results for nonlinear quadratic integral equations of fractional order in Banach algebra," Fractional Calculus and Applied Analysis, vol. 16, no. 4, pp. 816-826, 2013.

[10] R. I. Avery and J. Henderson, "Two positive fixed points of nonlinear operators on ordered Banach spaces," Communications on Applied Nonlinear Analysis, vol. 8, no. 1, pp. 27-36, 2001.

[11] R. W. Leggett and L. R. Williams, "Multiple positive fixed points of nonlinear operators on ordered Banach spaces," Indiana University Mathematics Journal, vol. 28, no. 4, pp. 673-688, 1979. 


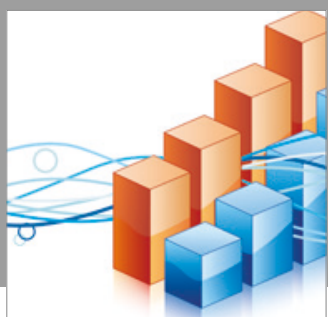

Advances in

Operations Research

vatersals

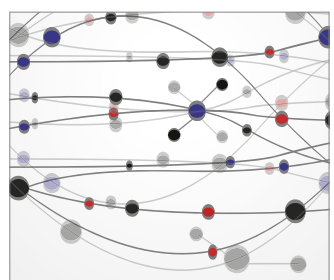

\section{The Scientific} World Journal
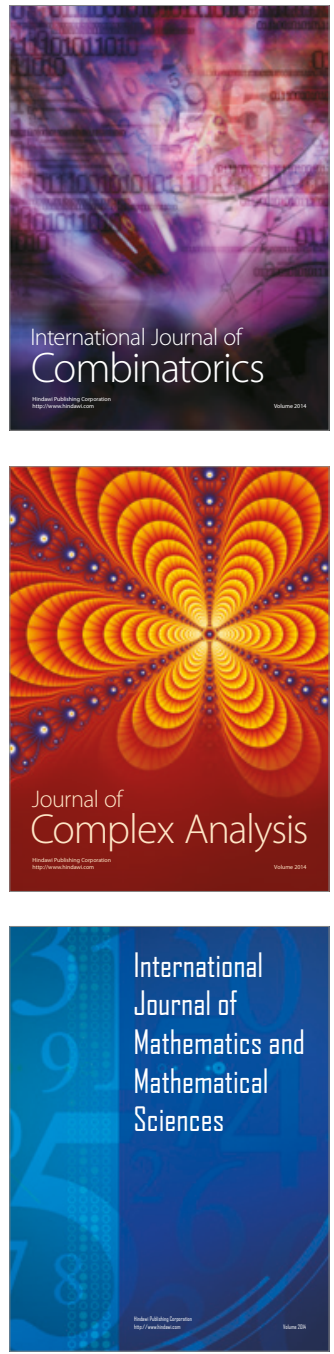
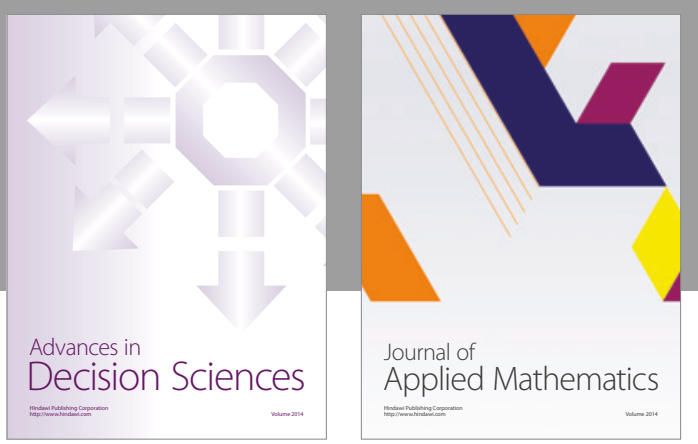

Algebra

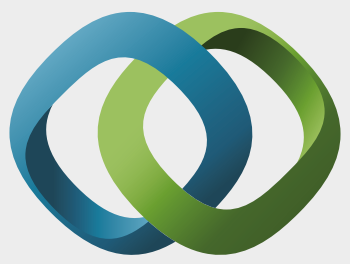

\section{Hindawi}

Submit your manuscripts at

https://www.hindawi.com
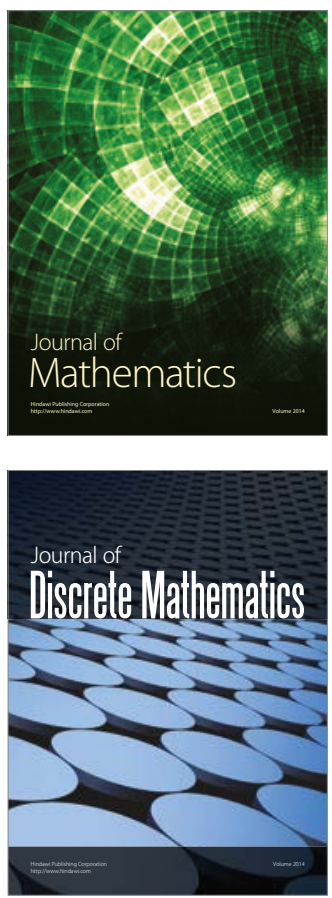

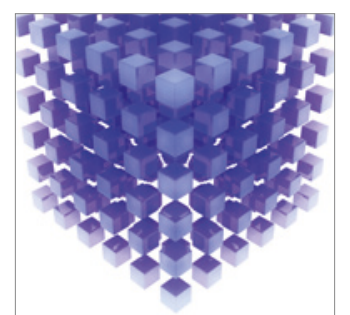

Mathematical Problems in Engineering
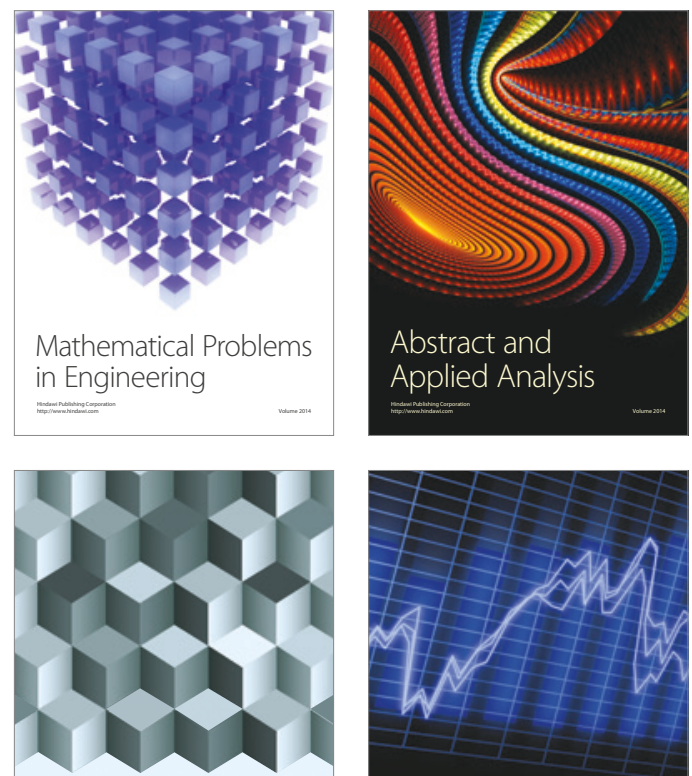

Journal of

Function Spaces

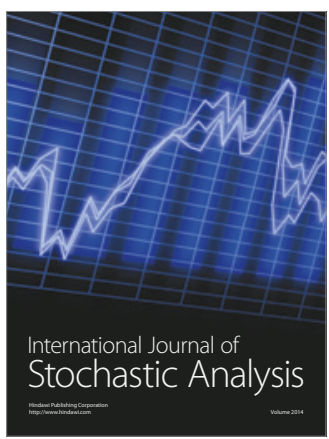

Probability and Statistics
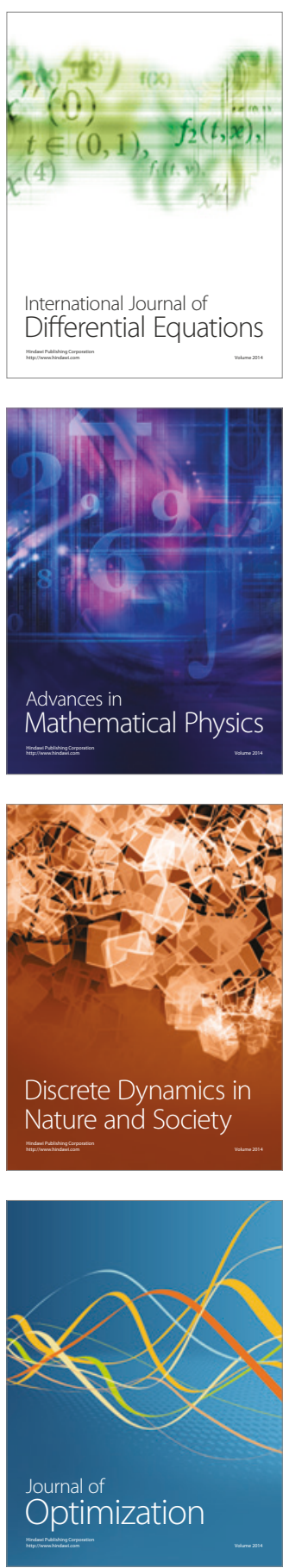